\title{
Televisiones universitarias en la República del Ecuador: estado de la cuestión en la emisión en analógico y vía web
}

\section{Roberto Freire Andino}

\section{Recibido: 14/08/2015}

Aceptado: 9/09/2015

\section{Resumen}

El campo comunicativo mundial está cambiando vertiginosamente, vemos cada día como los medios y las tecnologías de comunicación juegan un papel estratégico y decisivo en la trasmisión de la información. La televisión por internet es uno de esos avances. La mayoría de los canales de televisión convencionales han incorporado difusión de contenidos por medio de la web al igual que las universidades. Se analiza la existencia de televisión analógica y digital en las universidades ecuatorianas. Se estudia los elementos generales y contenidos de los canales de televisión universitaria por internet. Se analiza la web universitaria a partir de tres tipos de tablas que presentan elementos de carácter formal y de contenido. También se analiza la plataforma YouTube a partir de una ficha tipo. Con los datos obtenidos de las cuatro tablas se presentan los resultados a modo de gráficos, para llegar a las conclusiones del estudio.

\section{Palabras clave}

Televisión Universitaria, Ecuador, YouTube, Audiovisual, Información audiovisual

\section{University TVs in the Republic of Ecuador: state of the art in analog and broadcast via web}

\section{Abstract}

The worldwide communications field is changing rapidly, we see each day as the media and communication technologies play a strategic and decisive role in the transmission of information. Internet TV is one of those advances. Most conventional television channels have built distributing content via the web as well as universities. The existence of analogue and digital TV in Ecuadorian universities analyzed. The general elements and content of television channels online university is studied. University web is analyzed from three types of tables that present formal elements and content. The YouTube platform is also analyzed from a standard card. With data from four tables the results are presented as graphs, to reach conclusions.

\section{Keywords}

University televisión, Ecuador, YouTube, Audiovisual, Audiovisual information

http://dx.doi.org/10.5209/rev CDMU.2015.v26.50631 


\section{INTRODUCCIÓN}

La televisión universitaria ha sido una utopía de las diferentes universidades que han visto coartado su proyectos por todo lo implica el montaje de un canal de televisión, su financiamiento, producción de programas, mantener una parrilla de programación que esté acorde con el debate universitario. En muchos caso cuando la universidad ha logrado tal cometido su alcance ha sido local.

A nivel mundial existe una transformación de paradigmas respecto de la televisión, el cambio de lo analógico a lo digital ha marcado una nueva época. La televisión ya no solo se mira en las pantallas destinadas para ese fin si no que ha migrado a internet.

Como lo afirma Martínez (2012) La televisión por internet es una nueva forma de ver y entender la televisión, en la cual la transmisión de imágenes se realiza a través de la red de Internet, así el ciberespacio permite la convergencia digital, materializada como la presencia de la televisión en Internet convirtiendo la red en un medio audiovisual, está generando un nuevo escenario que ofrece retos, pero también atractivas posibilidades.(Romero López 2012)

La combinación de TV e internet busca dar un paso pionero de convergencia entre dos medios para sumar la masividad del medio televisivo con la interactividad de la web, y el impacto estético de la pantalla de la televisión con la información personalizada en el PC de los usuarios; la fuerza narrativa televisiva con las múltiples posibilidades del relato web. (Romero López 2012)

El contexto internacional, nos permite tener una visión amplia de la televisión universitaria, los diferentes aporte que han dado los canales universitarios a la televisión por internet, así tenemos al canal de UNAM (http://www.tvunam.unam.mx/) creado en el año 2005, que a decir de la ATEl es una de los mejores en Iberoamérica, otro ejemplo es el de la televisión por Internet en la Universidad de California (http://www.uctv.tv/) creado en el año 2000.

Son dos ejemplos que representan un modelo a imitar ya que en la actualidad poseen grandes audiencias al igual que cualquier televisión convencional. Disponen de parrilladas de programación que cubre casi las 24 horas del día, se puede apreciar toda clase de documentales, culturales, científicos, que pueden ser consultados sin restricciones.

La televisión universitaria por internet no solo se queda en su URL, también difunde sus contenidos a través de la diferentes plataformas de difusión de contenidos como es el caso de YouTube, que en muchos casos se convierte en el canal de la universidad, este canal se convierte en el medio de difusión oficial de los centros de educación, que a través de la red social divulgan sus diferentes actividades, científicas, académicas, culturales.

\section{SITUACIÓN EN ECUADOR}

Hablar de la televisión universitaria en Ecuador es remitirse a la televisión analógica, en un recuento breve de la historia se puede identificar que para el año de 1975 la Universidad Católica de Cuenca compra el canal 3 llamado "Ciudad de Cuenca", para 1983 el canal cambia de nombre a llamarse "Telecuenca Canal Universitario Católico", esto supondría un desarrollo de la televisión universitaria, pero no sucede, 23 años después (2006) 
la Universidad Técnica del Norte inicia transmisiones en su canal universitario, un año después la Universidad Católica Santiago de Guayaquil tiene su sistema de radio y televisión.

La televisión universitaria por internet no varia mucho ya que los centros de educación antes mencionados en dos de los tres casos pasan su parrilla de programación análoga a la web. La Universidad Católica Santiago de Guayaquil crea su canal digital en el año 2013, la Universidad San Francisco de Quito crea su canal digital en el año 2010, por lo que se puede decir que la televisión universitaria en internet en el Ecuador es relativamente joven.

Otros centros de educación superior utilizan diversas herramientas en la web para la difusión de sus actividades especialmente para la transmisión en vivo de sus eventos académicos, culturales, políticos o deportivos. El canal más utilizado por estos centros es el Ustream, que sirve para la difusión en directo de una actividad. Aquí no se encuentra ningún repositorio de archivos.

\section{OBJETIVOS Y METODOLOGÍA}

Los objetivos que nos hemos marcado en el presente trabajo son los siguientes

1. Determinar las universidades ecuatorianas que tienen televisión universitaria

2. Identificar los contenidos distribuidos a través de las televisiones universitarias

3. Indagar el uso de la red social YouTube como canal digital en las universidades ecuatorianas

4. Analizar globalmente la situación de la comunicación digital de las universidades ecuatorianas

El trabajo se realiza a través de un análisis de contenido manual una metodología de análisis de comunicación de forma sistemática, objetiva, con el fin de medir determinadas variables. (Kerlinger citado por Wimmer, 2001: 135) Para el análisis se emplea tres tablas que contienen un total de 22 variables, las cuales permiten obtener las propiedades visibles de las televisiones universitarias ecuatorianas y del canal YouTube de los centros de educación superior. Para establecer estas variables nos hemos basado en:

1. Adaptar la tabla establecida por Murillo y Caldera (2014) para crear dos tablas una de los aspectos generales y otra destinada a la televisión universitaria en la web.

2. Ampliar la tabla propuesta por Barredo (2013) para el análisis de YouTube, ya que el canal presta diferentes variables que permiten un análisis más competo de esta red social.

La muestra escogida son las 54 universidades del Ecuador de las categorías A, B, C, D ${ }^{1}$. La fuente principal para el acceso a las universidades del Ecuador ha sido la sede web del Consejo de Educación Superior del Gobierno de Ecuador (http://www.ces.gob.ec/ies/universidades-y-escuelas-politecnicas) en donde se puede localizar el directorio completo de las universidades tanto de carácter público, privado y cofinanciadas ${ }^{2}$, contando con un

1

Categorías de las universidades y politécnicas ecuatorianas otorgada por el CEAACES en el año 2013.

2

Financiamiento económico público y privado. 
total de 54 universidades y politécnicas en Ecuador. No se considera en éste trabajo a las universidades Yachay, UNAE, De las Artes e Ikian que son de reciente creación.

La recolección de la información se realizo entre el mes de enero y febrero del 2015.

El listado de las universidades y politécnicas públicas, privadas y cofinanciadas se presenta a continuación de acuerdo a su categoría:

\section{Categoría A}

Escuela Politécnica Nacional

Escuela Superior Politécnica del Litoral

Universidad San Francisco de Quito

Facultad Latinoamericana de Ciencias Sociales

Universidad Andina Simón Bolívar

Universidad de las Fuerzas Armadas - ESPE

\section{Categoría B}

Escuela Superior Politécnica de Chimborazo

Pontificia Universidad Católica del Ecuador

Universidad Casa Grande

Universidad Católica de Santiago de Guayaquil

Universidad Central del Ecuador

Universidad de Cuenca

Universidad del Azuay

Universidad Estatal de Milagro

Universidad Nacional de Loja

Universidad Particular Internacional SEK

Universidad Politécnica Salesiana

Universidad Técnica de Ambato

Universidad Técnica del Norte

Universidad Técnica Estatal de Quevedo

Universidad Técnica Particular de Loja

Universidad Tecnológica Empresarial de Guayaquil

Universidad Tecnológica Equinoccial

Universidad Tecnológica Indoamérica

Universidad de los Hemisferios

Universidad Estatal Amazónica www.epn.edu.ec

www.espol.edu.ec

www.usfq.edu.ec

www.flacso.org.ec

www.uasb.edu.ec

www.espe.edu.ec

www.espoch.edu.ec

www.puce.edu.ec

www.casagrande.edu.ec

www.ucsg.edu.ec

www.uce.edu.ec

www.ucuenca.edu.ec

www.uazuay.edu.ec

www.unemi.edu.ec

http://unl.edu.ec/

http://www.uisek.edu.ec/\#

www.ups.edu.ec

www.uta.edu.ec

http://www.utn.edu.ec/

http://www.uteq.edu.ec/

http://www.utpl.edu.ec/

http://www.uteg.edu.ec/

http://www.ute.edu.ec/

http://www.uti.edu.ec/

http://www.uhemisferios.edu.ec/

http://www.uea.edu.ec/ 


\begin{tabular}{|c|c|}
\hline Universidad Politécnica del Carchi & http://www.upec.edu.ec/ \\
\hline Universidad Iberoamericana & http://www.unibe.edu.ec/ \\
\hline Instituto de Altos Estudios Nacionales & http://iaen.edu.ec/ \\
\hline \multicolumn{2}{|l|}{ Categoría C } \\
\hline Escuela Superior Politécnica Agropecuaria de Manabí & http://www.espam.edu.ec/ \\
\hline Universidad de Especialidades Turísticas & http://www.uct.edu.ec/ \\
\hline Universidad de las Américas & http://www.udla.edu.ec/ \\
\hline Universidad del Pacífico Escuela de Negocios & http://upacifico.edu.ec/web/ \\
\hline Universidad Estatal de Bolívar & http://www.ueb.edu.ec/web1/ \\
\hline Universidad Internacional del Ecuador & $\begin{array}{l}\text { http://www.uide.edu.ec/SITE/inde } \\
\text { x.php }\end{array}$ \\
\hline Universidad Laica Vicente Rocafuerte de Guayaquil & http://www.ulvr.edu.ec/ \\
\hline Universidad Metropolitana & http://umet.edu.ec/ \\
\hline Universidad Nacional del Chimborazo & http://www.unach.edu.ec/ \\
\hline Universidad Particular de Especialidades Espíritu Santo & http://www.uees.edu.ec/ \\
\hline Universidad Regional Autónoma de los Andes & http://www.uniandes.edu.ec/ \\
\hline Universidad Técnica de Babahoyo & http://www.utb.edu.ec/ \\
\hline Universidad Técnica de Cotopaxi & http://www.utc.edu.ec/ \\
\hline Universidad Tecnológica Israel & http://uisrael.edu.ec/ \\
\hline Universidad Estatal Península de Santa Elena & http://www.upse.edu.ec/ \\
\hline Universidad Particular San Gregorio de Portoviejo & http://www.sangregorio.edu.ec/ \\
\hline Universidad Técnica de Manabí & http://www.utm.edu.ec/ \\
\hline Universidad Tecnológica ECOTEC & $\begin{array}{l}\text { http://www.universidadecotec.ed } \\
\text { u.ec/ }\end{array}$ \\
\hline \multicolumn{2}{|l|}{ Categoría D } \\
\hline Universidad Agraria del Ecuador & http://www.uagraria.edu.ec/ \\
\hline Universidad de Guayaquil & http://www.ug.edu.ec/ \\
\hline Universidad Estatal del Sur de Manabí & http://unesum.edu.ec/ \\
\hline Universidad Laica Eloy Alfaro de Manabí & http://www.uleam.edu.ec/ \\
\hline Universidad Técnica de Machala & $\begin{array}{l}\text { http://www.utmachala.edu.ec/por } \\
\text { talweb/ }\end{array}$ \\
\hline Universidad Técnica Luis Vargas Torres de Esmeraldas & http://utelvt.edu.ec/ws2/ \\
\hline Universidad Católica de Cuenca & http://www.ucacue.edu.ec/ \\
\hline Universidad de Otavalo & http://www.uotavalo.edu.ec/ \\
\hline
\end{tabular}

Tabla 1. Páginas de Universidades y Politécnicas de Ecuador 
Cada uno de los sitios web ha sido examinados de acuerdo a las siguientes tablas de análisis.

Aspectos Generales

\begin{tabular}{|c|c|c|c|c|c|}
\hline Institución & URL & $\begin{array}{l}\text { Naturaleza } \\
\text { jurídica }\end{array}$ & $\begin{array}{l}\text { Televisión en la } \\
\text { web }\end{array}$ & $\begin{array}{l}\text { Televisión } \\
\text { analógica }\end{array}$ & Canal YouTube \\
\hline $\begin{array}{l}\text { Se describe el } \\
\text { nombre de la } \\
\text { universidad }\end{array}$ & $\begin{array}{l}\text { Corresponde a la } \\
\text { localización de la } \\
\text { página web de la } \\
\text { universidad }\end{array}$ & $\begin{array}{l}\text { Para describir el } \\
\text { financiamiento } \\
\text { de la universidad } \\
1 \text { pública } \\
2 \text { privada } \\
3 \text { cofinanciada }\end{array}$ & $\begin{array}{l}\text { Mostrar si la } \\
\text { institución } \\
\text { cuenta con una } \\
\text { televisión digital } \\
1 \mathrm{Si} \\
2 \mathrm{No}\end{array}$ & $\begin{array}{l}\text { Mostrar si la } \\
\text { institución } \\
\text { cuenta con una } \\
\text { televisión } \\
\text { analógica } \\
1 \text { Si } \\
2 \mathrm{No}\end{array}$ & $\begin{array}{l}\text { Identificar si la } \\
\text { institución } \\
\text { cuenta con un } \\
\text { canal YouTube } \\
1 \mathrm{Si} \\
2 \mathrm{No} \\
3 \text { cerrado }\end{array}$ \\
\hline
\end{tabular}

Tabla 2: Aspectos generales de la televisión universitaria.

La tabla inicia con los datos de identificación de la universidad: el nombre de la universidad, su URL y su naturaleza jurídica. Al ingreso al sitio de web el nombre de la universidad se identifica en la parte superior.

La naturaleza jurídica de la universidad nos indica si se trata de una universidad pública, privada o cofinanciada, el dato se extrae de la página web del CES (Consejo de Educación Superior) del Gobierno del Ecuador.

El siguiente dato en la tabla es ver si la universidad cuenta con televisión analógica y/o televisión digital (presencia en la web); analizando si además cuenta con canal YouTube. La televisión analógica de la universidad no fue objeto de estudio para el presente artículo.

Si la universidad cuenta con un canal con presencia en la web se lo analiza con la siguiente tabla.

\begin{tabular}{|c|c|c|c|c|c|c|c|c|c|}
\hline Institución & $\begin{array}{l}\text { Canal } \\
\text { Digital URL }\end{array}$ & $\begin{array}{l}\text { Año } \\
\text { creación }\end{array}$ & $\begin{array}{l}\text { Año de } \\
\text { emisión }\end{array}$ & Emisión & $\begin{array}{l}\text { Vídeos } \\
\text { recientes en la } \\
\text { página } \\
\text { principal. }\end{array}$ & $\begin{array}{l}\text { Contiene } \\
\text { repositorio de } \\
\text { vídeos }\end{array}$ & $\begin{array}{l}\text { Secciones del } \\
\text { canal }\end{array}$ & $\begin{array}{l}\text { Contenido de los } \\
\text { vídeos }\end{array}$ & $\begin{array}{l}\text { Datos } \\
\text { contacto }\end{array}$ \\
\hline $\begin{array}{l}\text { Se } \\
\text { describe } \\
\text { el nombre } \\
\text { de la } \\
\text { universida } \\
\text { d }\end{array}$ & $\begin{array}{l}\text { Correspond } \\
\text { e a la } \\
\text { dirección } \\
\text { del canal } \\
\text { digital de la } \\
\text { universidad }\end{array}$ & \begin{tabular}{l}
\multicolumn{2}{c}{ Se determina la } \\
fecha de \\
creación del \\
canal
\end{tabular} & $\begin{array}{l}\text { Se } \\
\text { identifica } \\
\text { el año de } \\
\text { inicio de } \\
\text { las } \\
\text { trasmisio } \\
\text { nes. }\end{array}$ & $\begin{array}{l}\text { Se describe } \\
\text { si sus } \\
\text { emisiones } \\
\text { son en: } \\
\text { 1. Directo } \\
\text { 2. Diferido } \\
\text { 3. Ambas }\end{array}$ & $\begin{array}{l}\text { Se identifica si } \\
\text { el canal digital } \\
\text { sube vídeos } \\
\text { periódicament } \\
\text { e } \\
\text { 1. Si } \\
\text { 2. No }\end{array}$ & $\begin{array}{l}\text { Se determina si } \\
\text { el canal cuenta } \\
\text { con archivo de } \\
\text { vídeos. } \\
\text { 1. Si } \\
\text { 2. No }\end{array}$ & $\begin{array}{l}\text { Muestra de } \\
\text { forma clara las } \\
\text { secciones que } \\
\text { tiene el canal } \\
\text { 1. Vídeos } \\
\text { Destacados } \\
\text { 2. Motor de } \\
\text { búsqueda } \\
\text { 3. Directorio }\end{array}$ & $\begin{array}{l}\text { Presentación de } \\
\text { los contenidos } \\
\text { de los canales } \\
\text { 1. Informativos } \\
\text { 2. Institucionales } \\
\text { 3. Educativos } \\
\text { 4. } \\
\text { Entretenimiento } \\
\text { 5. Divulgación } \\
\text { 6. Culturales } \\
\text { 7. Otros }\end{array}$ & $\begin{array}{l}\text { Descripción de } \\
\text { los datos } \\
\text { referenciales } \\
\text { del canal } \\
\text { 1. Correo-e } \\
\text { 2 Dirección } \\
\text { 3. Teléfono } \\
\text { 4.sin datos }\end{array}$ \\
\hline
\end{tabular}

Tabla 3: Canales digitales de las universidades y politécnicas ecuatorianas.

La tabla inicia con los datos de identificación del canal en la web: el nombre de la universidad, la dirección URL del canal en la web, también se ubica el año en el que fue fundado, y el año en el que inician su transmisión en el caso que ofrecieran dichos datos.. Al ingreso al sitio de web el nombre de la universidad y del canal se identifica en la parte superior. 
Los siguientes datos en la tabla son de interés para el objeto de estudio, los que se analizarán para conseguir los resultados a ser expuestos en las conclusiones.

En las diferentes televisiones se busca si sus emisiones son en directo o en diferido, también indagamos si la pagina principal del canal ofrece vídeos recientes y/o si contiene repositorios de vídeos.

La siguiente columna hace referencia a si la página cuenta con algunos elementos que ayudan a los usuarios a la hora de navegar en la pagina web del canal; se examina si presentan vídeos destacados, motor de búsqueda, directorio de contenidos.

El siguiente apartado busca averiguar los tipos de contenido de los vídeos que se pueden visualizar en los canales de las televisiones universitarias.

La última columna se plantean los datos de contacto del canal como correo electrónico, número de teléfono.

Algunas universidades aún sin contar con una televisión como tal, poseen su canal en la plataforma YouTube, no son exactamente un canal de televisión en su concepto establecido, pero sirven para la difusión de diferentes contenidos en forma de vídeos colgados en la red. La plataforma YouTube también forma parte del objeto de estudio.

Para el análisis de la plataforma YouTube de las universidades ecuatorianas se utiliza la siguiente tabla.

\begin{tabular}{|c|c|c|c|c|c|c|c|c|c|}
\hline Institución & URL & $\begin{array}{l}\text { Fecha de } \\
\text { Creación }\end{array}$ & $\begin{array}{l}\text { Número } \\
\text { suscripciones }\end{array}$ & de & $\begin{array}{l}\text { Número } \\
\text { visualizaciones } \\
\text { globales }\end{array}$ & $\begin{array}{l}\text { Número de } \\
\text { vídeos }\end{array}$ & Canales & $\begin{array}{l}\text { Comenta } \\
\text { rios }\end{array}$ & $\begin{array}{l}\text { Último } \\
\text { vídeo } \\
\text { subido }\end{array}$ \\
\hline $\begin{array}{l}\text { Se describe el } \\
\text { nombre de la } \\
\text { universidad }\end{array}$ & $\begin{array}{l}\text { Corresponde a la } \\
\text { dirección del } \\
\text { canal YouTube de } \\
\text { la universidad }\end{array}$ & $\begin{array}{l}\text { Se indica } \\
\text { cuando fue } \\
\text { creado el } \\
\text { canal } \\
\text { YouTube }\end{array}$ & $\begin{array}{l}\text { Cantidad } \\
\text { suscriptores } \\
\text { canal }\end{array}$ & $\begin{array}{l}\text { de } \\
\text { del }\end{array}$ & $\begin{array}{l}\text { Cantidad total } \\
\text { visualizaciones que } \\
\text { ha tenido el canal }\end{array}$ & $\begin{array}{l}\text { Cantidad de } \\
\text { vídeos que } \\
\text { tiene el canal }\end{array}$ & $\begin{array}{l}\text { Se describe cuantos } \\
\text { canales de YouTube } \\
\text { ha compartido }\end{array}$ & $\begin{array}{l}\text { Cantidad } \\
\text { de } \\
\text { comentar } \\
\text { ios que } \\
\text { tiene el } \\
\text { canal }\end{array}$ & $\begin{array}{l}\text { Tiempo } \\
\text { de la } \\
\text { última } \\
\text { actividad } \\
\text { realizada } \\
\text { en } \\
\text { YouTube }\end{array}$ \\
\hline
\end{tabular}

Tabla 4: YouTube de las universidades y politécnicas ecuatorianas

La tabla inicia con los datos de identificación del canal en YouTube: el nombre de la universidad, la dirección URL del canal en YouTube, también se ubica el año en el que fue creado. Al ingreso al canal se analiza la existencia del nombre, logo de la universidad se encuentra en la parte superior izquierda, además de la fotografía de identificación.

El siguiente dato en la tabla describen la actividad que realizan las universidades en la plataforma describe la cantidad de seguidores, la cantidad de visualizaciones de los vídeos del canal, el total de vídeos subidos, la cantidad de comentarios que dejan las personas y un dato que se estima importante se describe el tiempo en el que se ha subido un vídeo en YouTube.

Las principales fuentes de información han sido las páginas web de las universidades ecuatorianas en sus diferentes categorías ya sean públicas, privadas o cofinanciadas y sus enlaces en sus propias sede web para su televisión o al canal YouTube. Se ha analizado a todas las universidades ecuatorianas, buscando, indagando la existencia de la televisión universitaria, su presencia en la web y el uso de otra plataforma para difundir contenidos inherentes a su institución. 


\section{RESULTADOS}

Más de la mitad de las universidades en Ecuador son Públicas, es decir que su financiamiento depende del estado, el $33 \%$ son autofinanciadas esto quiere decir que sus recursos provienen del sector privado y de los costos que por colegiatura y matriculas cobran a los estudiantes, una cuarta parte de las universidades son cofinanciadas, sus recursos provienen del estado, de aporte privado y de la colegiatura y matricula que pagan los estudiantes.

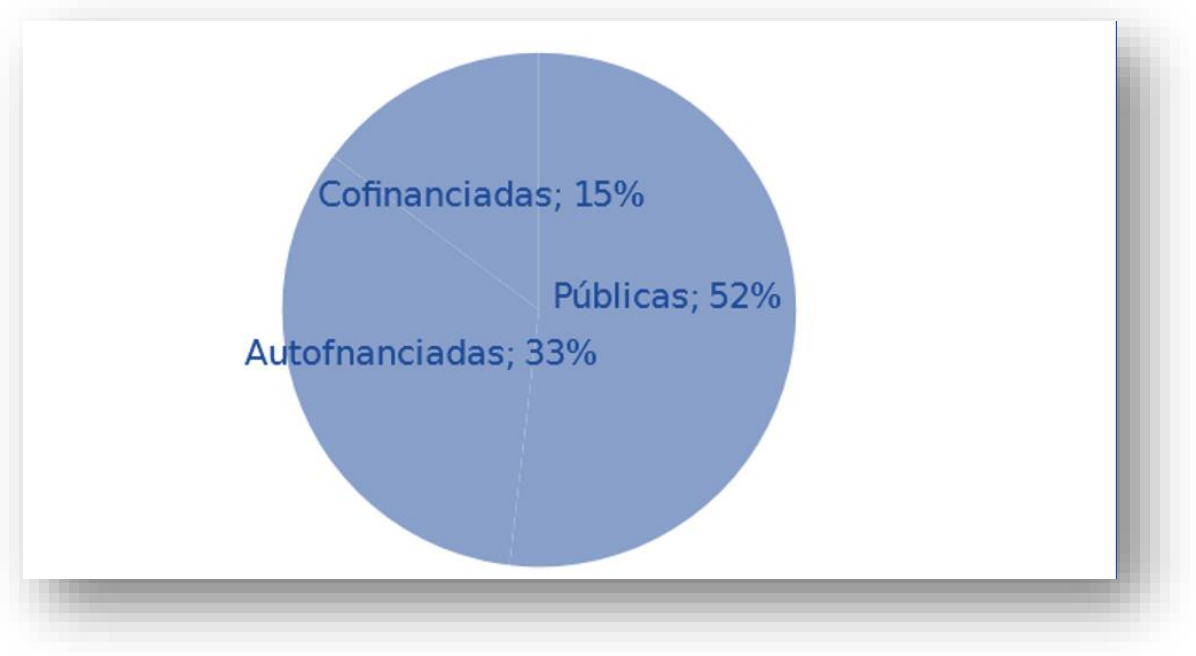

Financiación de las universidades

Destaca la baja cantidad de televisiones universitarias en el Ecuador, tan solo el $25 \%$ de las universidades cuentan con esta herramienta de apoyo a la educación, mientras que el $75 \%$ de las universidades no cuentan con televisión universitaria en la web. El 57\% de la televisión universitaria corresponden a las universidades públicas, las universidades privadas con el $21.5 \%$ al igual que las universidades cofinanciadas.

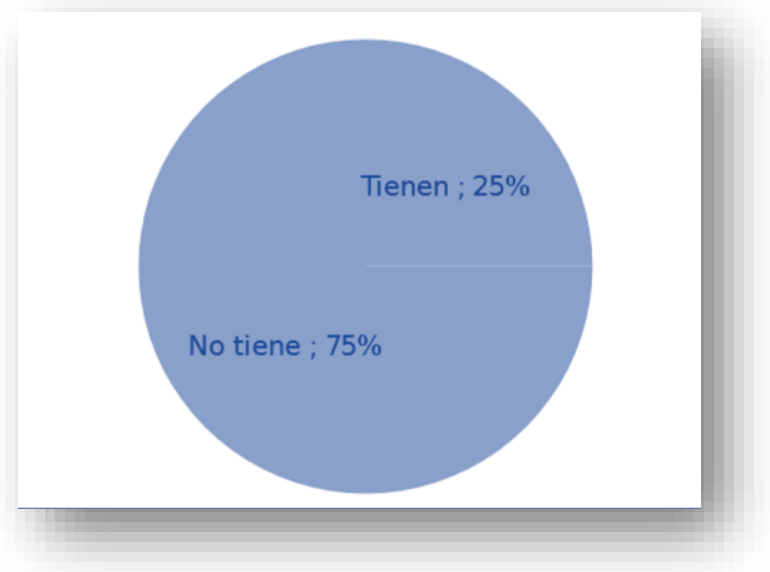

Universidades Ecuatorianas 
Las universidades que tiene un canal analógico en señal abierta es el 7.14\%, las restantes no cuentan con una herramienta de difusión de sus actividades, logros y puedan ser conocidos por la comunidad.

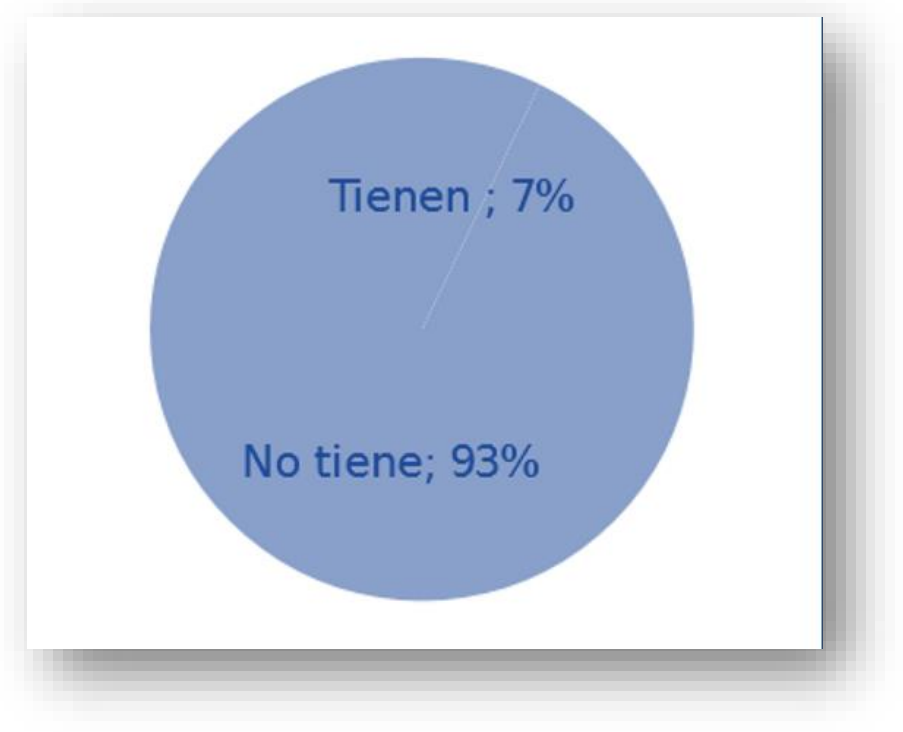

Televisión Analógica

El 72\% de las televisiones universitarias en la web cuentan con emisiones en directo. Se debe precisar que estas emisiones no son constantes durante las 24 horas, algunas de ellas que tienen el canal analógico transfieren su programación a su canal en la web. No se cuenta con una parrilla de programación estable. En otras la transmisión en directo es de eventos conmemorativos de la institución que no requieren de producción.

Las emisiones en diferido de las televisiones universitarias públicas, privadas y cofinanciadas es de $90 \%$.

Los aspectos formales en la televisión universitaria ecuatoriana en porcentajes medios, ofrecen elementos para el fácil uso de la web televisiva, que se describen a continuación: vídeos recientes, vídeos destacados, motor de búsqueda, directorio de vídeos o repositorio de vídeos; otras no cuentan con los elementos antes descritos dificultando el uso de estas herramientas a los consumidores de la televisión.

Todas las universidades de la categoría A, que cuentan con un canal de televisión en la web tienen programas educativos, que es lo apropiado ya que esto es la base de la televisión educativa, el $50 \%$ de las universidades de ésta categoría tienen otros tipos de programación diversificándose entre institucionales, de entretenimiento y divulgación.

Llama la atención que el $77 \%$ de las universidades de la categoría B no tengan programación educativa, lo que se puede entender es que mantienen programas de diferente variedad que no requiere de mucha producción, algunas de ellas tan solo se remiten a la transmisión de los eventos institucionales como aniversarios institucionales, inauguraciones de juegos deportivos entre otros. 
Los contenidos informativos, de entretenimiento y divulgativos/culturales se encuentran en un $47 \%$ de las televisiones universitarias de la categoría A, y en un $56 \%$ en las televisiones universitarias de la categoría B analizadas.

\section{Televisión universidades categoría A Televisión universidades categoría B}
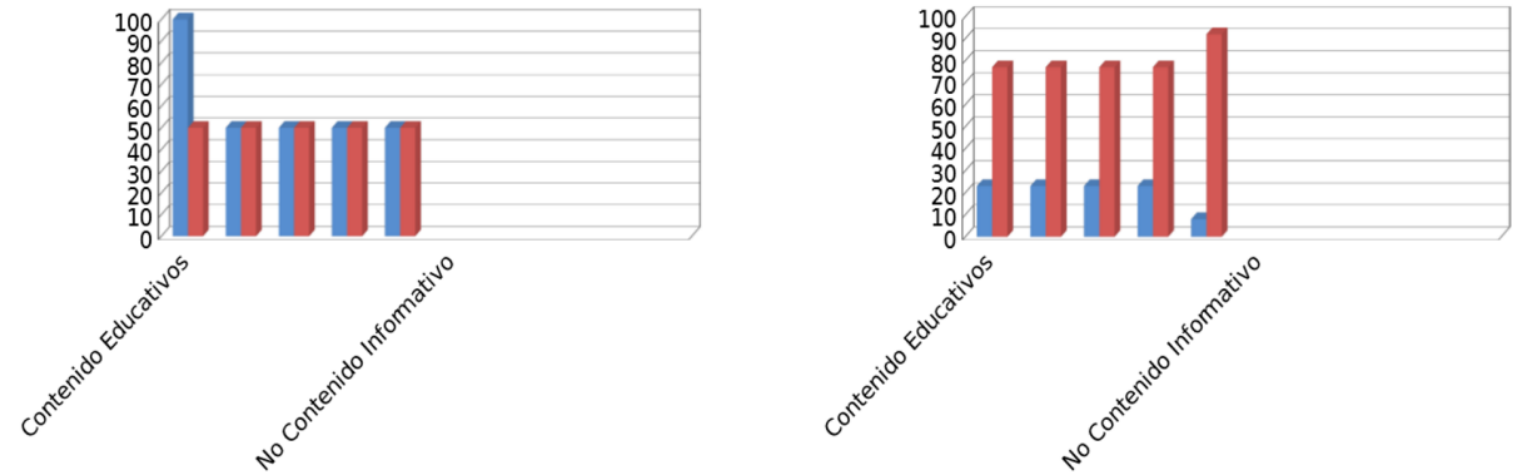

Las televisiones universitarias de las categorías C y D su programación es más homogénea en los contenidos que presentan sus porcentajes son muy semejantes, se puede destacar en $47 \%$ se encuentra programas educativos e institucionales, otro $47 \%$ de los programas son de entretenimiento, divulgación - cultural

\section{Televisión universidades categoría C Televisión universidades categoría D}
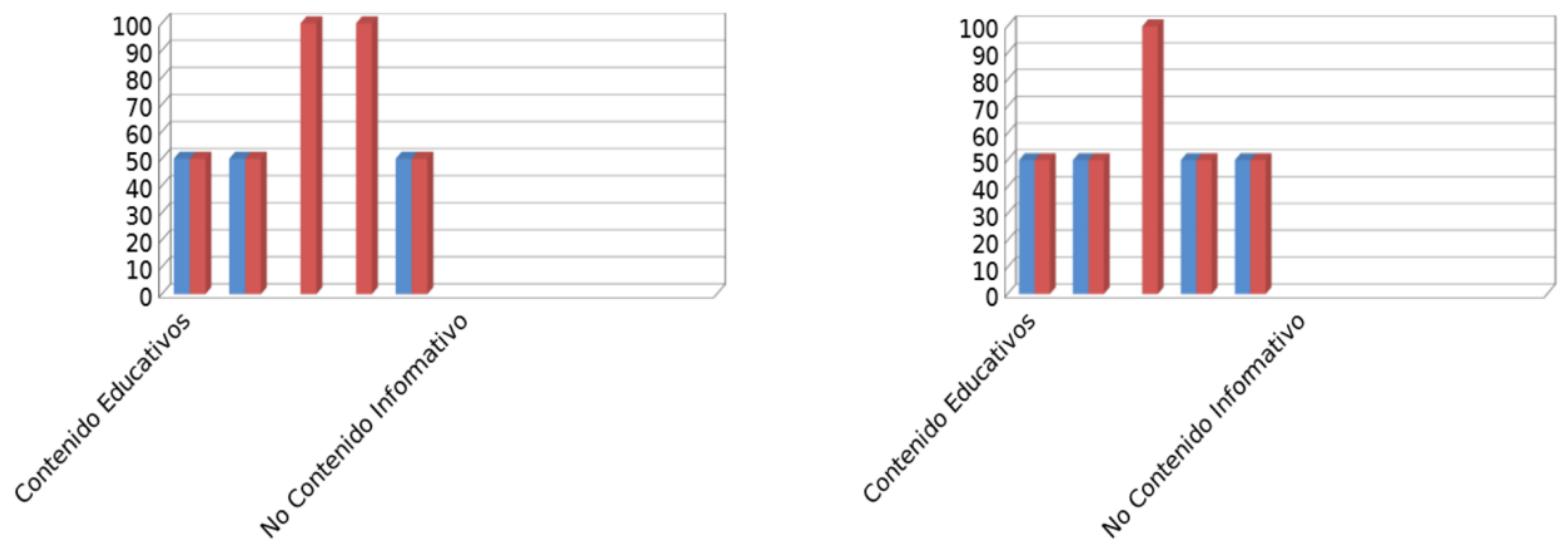


\section{YOUTUBE}

Las universidades ecuatorianas que tienen presencia en la plataforma YouTube es de un 91\%, el estar en este canal requiere de menos medios para su producción y difusión, también ofrece menos posibilidades que un canal en una sede web convencional. La presencia de las universidades en éste canal es desde el año 2006, y un gran número de universidades tiene su apertura en el año 2014. Algunos centros de educación han cerrado YouTube por el cambio de administraciones (Rectores) y han abiertos otro en la misma plataforma.

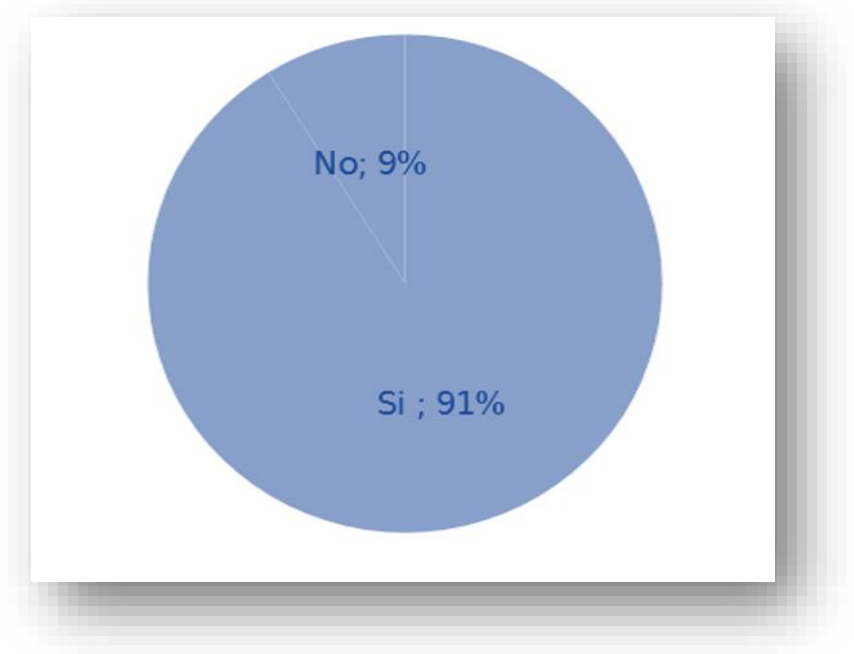

El número de suscriptores en el canal YouTube de las universidades de la categoría A, se encuentran por debajo de las 1000 suscripciones tan solo una universidad tiene 22474 seguidores. En la universidades de categoría B las suscripciones son mucho menor se encuentra por debajo de los 1000 y su variación se encuentra entre 4 a 988, tan solo una universidad en ésta categoría llega a 3652 suscripciones. En la universidades de la categoría C y $D$ las suscripciones a sus canales institucionales en la plataforma YouTube se encuentran por debajo de los 850 seguidores.

El número de visualizaciones globales que se encuentra en las universidades no están relacionadas en directa proporción con los vídeos que tiene en su canal así se puede ver universidades con menos cantidad de vídeos tiene mayor número de visualizaciones, esto se produce por la "imagen corporativa" que tiene algunas universidades frente a otras.

Llama la atención la casi nula cantidad de comentarios que tiene los canales YouTube de las universidades ecuatorianas, estos no se encuentran en directa relación con la cantidad de visualizaciones de los vídeos, así podemos ver que existe canales que sus vídeos han sido reproducidos sobre el medio millón de veces y no poseen un solo comentarios, tan solo una universidad que tiene sobre los ocho millones de reproducciones tenga 137 comentarios, el resto de universidades se encuentran entre cero y once. 
La plataforma YouTube permite compartir otros canales en el mismo entorno web, sin embargo las universidades ecuatorianas utilizan muy poco esta herramienta las universidades de la categoría B que utilizan YouTube son $36 \%$ son las que mas comparten con otros canales.

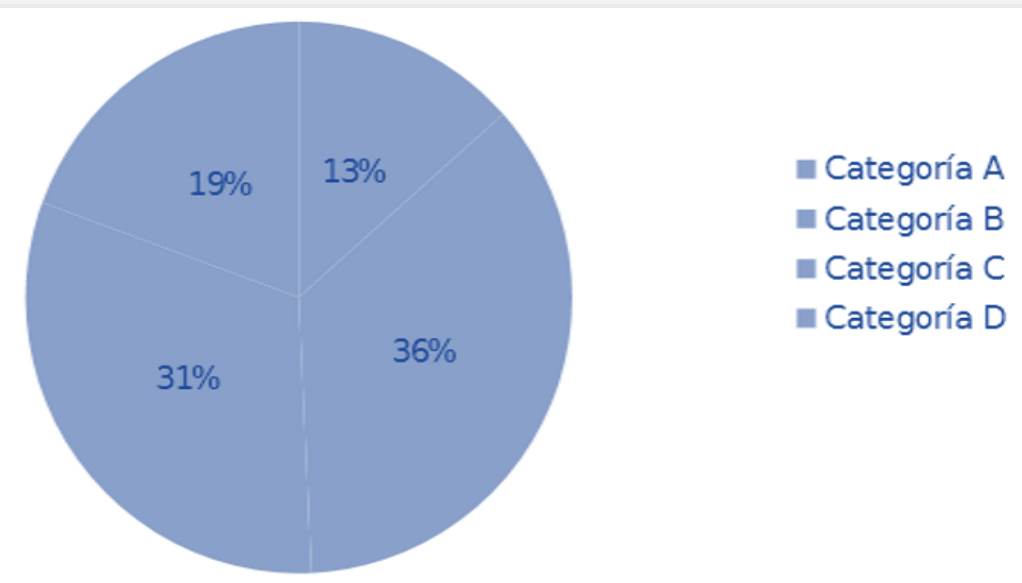

La actividad de las universidades en la red es variada se encuentran canales que suben con periodicidad sus vídeos entre una semana a un mes, mientras que otras universidades se olvidaron de esta herramienta ya que se muestra su inactividad desde el mismo año que le crearon, algunas universidades no han tenido actividad en la plataforma por seis años, parecen haber olvidado y abandonado su cuenta en esta interactiva red de intercambio de vídeos.

\section{CONCLUSIONES}

A la televisión se le mira desde diferentes aristas, como un aparato de poder, fuente de información entre otras, y se le utiliza para diferentes fines desde generar opinión, pasando como un instrumento de venta y para educar. Las universidades no han dejado pasar la oportunidad de utilizar a éste medio para comunicarse con la comunidad y difundir sus diversas actividades a los diferentes actores y sectores sociales.

La potencialidad de la televisión tan solo ha sido utilizada por muy pocas universidades en el Ecuador, existe una resistencia por parte de muchas para implementar una televisión universitaria.

Las instituciones de educación superior en el Ecuador, de acuerdo a los resultados precedentes, se encuentran escalonadas desde el punto de vista de la televisión universitaria por internet. La televisión universitaria en el Ecuador no siempre cumple con su encargo social, su uso no es el adecuado por parte de la institución, en algunos casos sirve para la venta de la marca e imagen de la institución.

Existen instituciones que han migrado su televisión análoga a la ciberesfera. Otras instituciones se mantienen en las redes sociales como Facebook, Twitter, YouTube, siendo la última la más utilizada para la divulgación de vídeos institucionales. 
Las universidades ecuatorianas casi en su totalidad cuentan con un canal institucional en la plataforma YouTube, el número de seguidores, como el número de vídeos publicados, no es un factor determinante para el número de visitas. Esto nos indica que la popularidad en la red se encuentra relacionada con los diferentes factores de calidad, mas no con el nombre de la institución. Las actividades en la red social por parte de muchos centros de educación superior son escaso algunos no han publicado nada desde su apertura, al contrario de otros que su actividad es periódica en algunos casos cada ocho días. La publicación de los vídeos no depende del presupuesto de las universidades se encuentran relacionado con los planes de comunicación y con los procesos de marketing de cada institución.

En esta foto fija de la televisión digital universitaria en el Ecuador se puede observar que aún queda un gran camino por recorrer para facilitar la información educativa y utilizar a la plataforma de difusión para los contenidos educativos.

Sin embargo de los dicho, en el Ecuador la televisión universitaria se encuentra en desarrollo, no se utiliza toda la pontencialidad en muchos de los casos los repositorios de los vídeos no se encuentra ubicados de acuerdo a la normativa para estos, le hace falta el tiempo de visionado, la definición del vídeo y más elementos informativos que permitan la usuario saber el tipo de material que tiene a disposición, en otros casos los repositorios de los vídeos se encuentra en el canal YouTube.

Es importante que se tome en cuenta las formas de organización televisivas existentes para la organización, sistematización en la difusión de los contenidos.

Es importante señalar el gran potencial de desarrollo de la televisión universitaria por internet, que en la generalidad de las universidades analizadas nos indican el desarrollo de unas, el letargo de otras y una gran mayoría que aún están buscando su desarrollo a través de políticas claras para su gestión.

Se trata de diseñar una televisión universitaria que no solo contemple la transmisión de información de los rectores de turno, sino que abarque la interacción académica en donde se pueda reconocer el avance de la ciencia y la tecnología y a los sujetos que la producen y que de cuenta de los diferentes paradigmas, estructuras de pensamiento propios de la universidad.

La televisión universitaria debe tener objetivos básicos, académicamente debe fomentar la base de la ciencia en una concepción epistemológica clara de acuerdo al contexto socio cultural del país, debe crear en el estudiante una consciencia de responsabilidad social que le permita percibir claramente sus responsabilidades y derechos; y culturalmente debe capacitar a los comunicadores para que entienda la multiplicidad de cosmovisiones. En síntesis, una televisión universitaria que tome en cuenta su sociedad su tiempo y su mundo.

Las universidades y los profesionales de la comunicación tienen la palabra. 


\section{REFERENCIAS BIBLIOGRÁFICAS}

Aguaded Gómez, J. Ignacio; Macías Gómez, Yolanda. “Televisión universitaria y servicio público”. Comunicar. Huelva, 2008, v. XVI, no 31, p. 681-689.

Barredo Ibáñez, Daniel (2013) La comunicación de las Universidades y Politécnicas ecuatorianas de clase A: un análisis comparativo de sus canales digitales http://www.revistalatinacs.org/13SLCS/2013_actas.html

Caldera - Serrano, Jorge (2003) La documentación audiovisual en las empresas. Biblios, núm 15, pp. 3 - 11

INEC (2015) www.ecuadorencifras.gob.ec/tecnologias-de-la-informacion-y-comunicacion-tic/

Gámiz Sánchez, V. y Romero López, A. (2012).La televisión universitaria, ele ejemplo de la Universidad de Granada .EDMETIC, Revista de Educación Mediática y TIC, 1 (1), 52-69.

Martínez Recio, A. y Conde, S. (2012).La televisión (por Internet) en la Universidad de Córdoba .EDMETIC, Revista de Educación Mediática y TIC, 1 (1), 29- 51.

http://www.telecuenca.com/webs/historia.php

Murillo Guerrero, María, Caldera-Serrano, Jorge (2014) Presencia y difusión de contenidos de las televisiones universitarias españolas en la web, Cuadernos de Documentación y Multimedia, Vol. 25. P. 25-38.

Pérez Agüera, José Ramón; Sánchez Jiménez, Rodrigo; Caldera - Serrano Jorge (2004). Adaptación de tecnologías stream y xml a centros de documentación en televisión. Revista Española de Documentación Científica, vol. 27, 441 - 454

Póveda - López, Inés Carmen; Caldera-Serrano, Jorge; Polo-Carrión, Juan-Antonio (2010). Definición del objeto de trabajo y conceptualización de los Sistemas de Información Audiovisual de la Televisión. Investigación Bibliotecológica, vol. 24, núm. 50, pp. 15 - 34

Wimmer R., Dominck J, (2001) Introducción a la Investigación de Medios Masivos de Comunicación, Thomson, México 